\title{
Le projet du care pour une résilience permanente de la ville. Une étude sur trois transects urbains dans le $5^{\mathrm{e}}$ arrondissement de Paris.
}

\author{
Designing care: towards a permanent resilience of the city.
}

\author{
Antonella Tufano ${ }^{1}$ \\ ${ }^{1}$ Ecole Nationale Supérieure d'Architecture de Paris La Villette, chercheur GERPHAU (EA 7486), \\ antonella.tufano@paris-lavillette.archi.fr
}

RÉSUMÉ. Les villes contemporaines sont concernées par des risques environnementaux (pic de chaleur ou de froid ; pollution...) qui touchent les populations les plus vulnérables. Dans ce cas, la résilience n'est pas seulement un processus de régénération s'activant a posteriori, mais pourrait s'inscrire dans une durée temporelle longue par un projet fondé sur la notion de care; le projet urbain se transformant en un système de projets et en fonctionnant selon des temporalités multiples, cette stratégie urbaine du care serait le garant d'une résilience permanente. La proposition avancée s'appuie sur des recherches conduites entre 2013 et 2017 dans un secteur du 5 e arrondissement de Paris à partir de l'observation des llots de Chaleur Urbains. Ce laboratoire urbain a permis de construire un système de projets, dont les effets varient en fonction de leur scalabilité (adaptation sectorielle à l'échelle micro et atténuation à l'échelle méso) et accompagnent une résilience urbaine constante et multi-partenariale.

ABSTRACT. In contemporary cities, natural and industrial risks are accompanied by an environmental risk (climatic, pollution...) that affects the most vulnerable populations. Designing care allows promoting permanent resilience which can mitigate the effects of these environmental risks. Designing care, the resilience is not only a process of recognition and regeneration built a posteriori, but a permanent awareness of the complexity of contemporary urbanity. This topic is based on research carried out between 2013 and 2017 in a sector of the 5th arrondissement of Paris. This urban laboratory has made it possible to construct a system of projects, by scaling and rescaling their effects in the urban life. These projects help to build a constant and multi-partnership resilience.

MOTS-CLÉS. ICU (Ilot de Chaleur Urbain), résilience urbaine, scalabilité, reliance, sollicitude.

KEYWORDS. Care, urban resilience, scaling/rescaling, Urban Heat Island (UHI).

\section{La résilience permanente : une stratégie de projets relationnels}

Dans les territoires exposés au risque, à la suite d'un événement majeur, la résilience (Walker, Salt, 2006 ; Dauphiné, Provitolo, 2007; Walker, Salt, 2012) paraît le moyen de rétablir une forme d'équilibre et de construire un discours permettant de surmonter le traumatisme vécu.

Cela implique une relation au temps du risque qui se fait dans un registre de l'a posteriori. Or, la notion de risque tend à investir les territoires d'une manière plus générale et, dans le contexte urbain, la question du risque environnemental reste difficile à définir et à cerner car des intérêts contradictoires se développent (Metzger, D’Ercole 2011 ; Metzger, Robert 2015). Le risque environnemental ordinaire, par exemple les variations du climat urbain en période de pic (froid ou chaleur), peuvent induire non seulement un sentiment d'inconfort, mais aussi des situations de précarité sanitaire ou d'exclusion sociale (Navarro-Carrascal, Michel-Gouillou, 2014).

A partir de ce constat et en nous appuyant sur une recherche qui s'est déroulée entre 2013 et 2017, dans le centre de la ville de Paris ( $5^{\mathrm{e}}$ arrondissement $)^{1}$, nous avons observé les modifications possibles

\footnotetext{
${ }^{1}$ L'objectif général de la recherche portait sur l'énergie à l'échelle du quartier et le rôle que le projet architectural et urbain pouvait jouer en tant que dispositif d'atténuation/adaptation dans un contexte urbain complexe faisant l'objet de plusieurs réglementations (au regard de l'énergie, de la notion de durabilité et du cadre patrimonial).Ces expériences de design des milieux urbains ont été conduites à l'Ecole Nationale Supérieure d'Architecture de Paris La Villette (recherche du Ministère de la Culture Ignis Mutat Res avec l'Università La Sapienza-Rome (N. Trasi mandataire, A. Tufano direction de l'équipe française : A. Brugnoni, informaticien, (c) 2017 ISTE OpenScience - Published by ISTE Ltd. London, UK - openscience.fr 
d'un quartier parisien à travers la problématique de l'Ilot de Chaleur Urbain (ICU) afin de comprendre si et en quoi les projets urbains et d'architecture pouvaient contribuer à la résilience urbaine. Dans le cadre de cette observation sur les ICU, la nécessité d'intégrer en amont une notion temporelle longue (Reghezza-Zitt, Rufat, 2015) et de créer les conditions d'une résilience permanente demandait une nouvelle forme de projet intégrant la notion relationnelle de care.

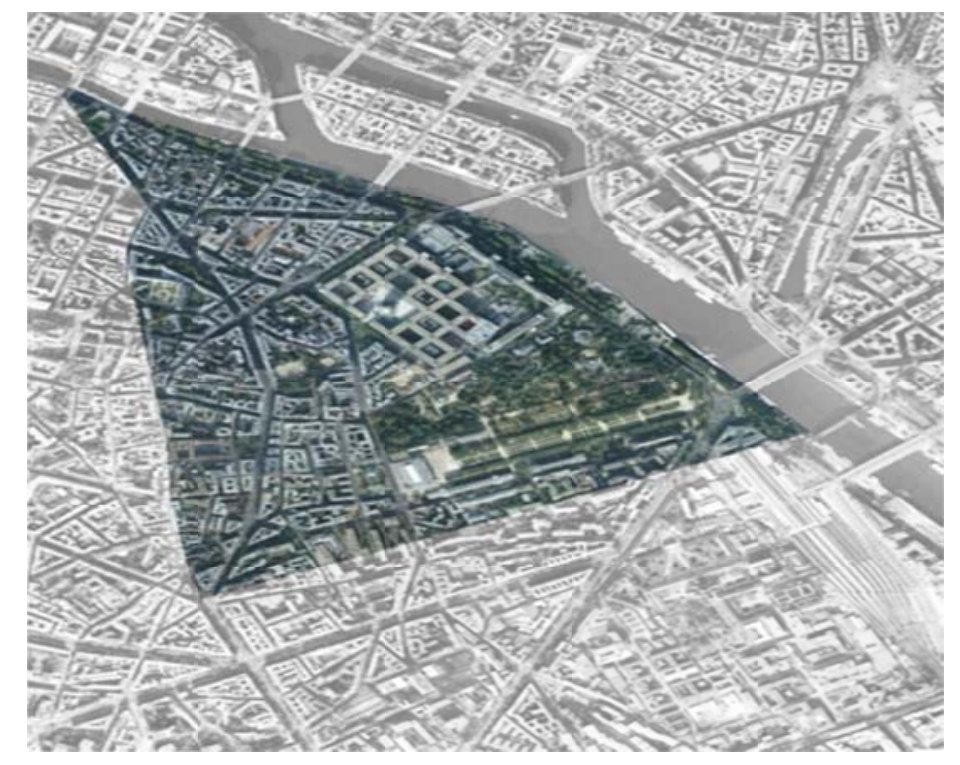

Figure 1. Périmètre du site d'étude à Paris 5e (Rue Monge, Rue Santeuil-rue Buffon, Bords de la Seine); sauf indication contraire, toutes les images ont été élaborées par l'équipe parisienne de la recherche Ignis Mutat Res à partir de la documentation mise à disposition par l'Atelier Parisien d"Urbanisme (APUR).

En introduisant la notion de care, nous proposons de déplacer la question de la résilience vers le traitement en amont par une série de projets permettant, d'une part, de prendre conscience d'un risque à travers des dispositifs de compréhension, partage et transmission de l'information et, d'autre part, de tenter une expérience de predicitive design, c'est-à-dire de favoriser par des propositions spatiales une gestion sobre qui accompagne la résilience permanente. Cette stratégie de projet donne forme à une constellation de solutions ; bien que les projets s'inscrivent dans les préconisations législatives concernant l'amélioration des performances énergétiques, nous avons tenté d'illustrer comment le projet peut être un levier de comportements individuels (Felli, 2014) facilitant la résilience. De surcroit, le renoncement des politiques vers les mesures qui ne sont pas classifiables, comme l'adaptation, nécessite une ouverture du débat à la société civile (Baudoui, 2014) et un empowerment citoyen vis-à-vis des questions de risque et de résilience (Wisner et alii, 2004 ; Cros et alii, 2010).

Le résultat espéré dans ce cadre de recherche était moins la construction d'un outil d'évaluation que l'observation critique d'un processus permettant d'inclure plusieurs variables et de montrer que l'activation différée dans le temps de certains micro-projets avait un impact comparable en matière d'amélioration des performances énergétiques et supérieur au regard de la capacité de résilience d'un territoire.

Le concepteur (de projets urbains, bâtiments, structures et mobiliers) est souvent démuni et le travail se fait de manière sectorielle posant la question de la séparation entre les objectifs prescriptifs à atteindre pour une ville durable et ceux, moins quantifiables mais plus dynamiques, de la résilience, combinant échelles et temporalités (Toubin, et al. 2012). Ainsi, sans prétention de réponse absolue ou de construction de modèles, nous avons creusé la piste des conditions opératoires pour une inversion de

ENSAD Nancy, O. Fatigato, architecte, ENSAPLV, M. Salerno, architecte, ENSAPM, S. Moreau et A. Pfizster ingénieurs ELEMENTS ingénierie); programme Confluences HeSAM avec le CNAM et l'ENSCI-Les Ateliers (A. Tufano coord.) ; Atelier Cycles Courts au sein du Centre Michel Serres pour l'Innovation (A. Tufano, J. Debrie coord.) ; ateliers interdisciplinaires IP 922 Energie 0, à l'ENSAPLV de 2013 à 2017 (dir. A. Tufano). 
la démarche de projet classique (Conan, 1990 ; Prost, 2014) et l'émergence d'un système de projets fondé sur l'urban caring.

L'hypothèse d'une relation entre des Ilots de Chaleur Urbains, les interventions visant la sobriété (réduction et/ou amélioration des performances énergétiques) et les comportements (individuels et urbains, notamment ceux qui pouvaient s'inscrire dans une logique du care) a été à la base de cette observation des projets et de l'identification d'une stratégie de projets relationnels qui pouvaient donner lieu à une résilience permanente. Il s'agit d'un travail empirique car la mise en place a priori d'un projet intégré (Terrin, 2015), ou mieux, complexe, demandait de nombreux franchissements d'échelles (des pratiques individuelles aux mécanismes normatifs trop peu incarnés) et il a été préférable de choisir un prisme d'observation -l'ICU- et regarder les relations complexes qui se tissaient et les seuils à franchir. L'ICU est un démonstrateur à l'échelle méso du système de projets qui permettrait de mettre en dialogue solutions spatiales et comportements, impact des matériaux et ressenti du climat, dispositif d'adaptation énergétique et effet sur les habitudes. Pour mettre en relation les paradoxes urbains (Marie, 2015) nous avons utilisé trois clés conceptuelles : la notion de care, inscrite dans le cadre urbain de la complexité et la transformation du projet en un système de projets.

\section{Prémisse théorique : le laboratoire urbain, un milieu habité}

\subsection{Urban caring}

La philosophe américaine Joan Tronto définit la société du care comme : « un ensemble complexe de pratiques, qui s'étendent de sentiments très intimes comme la pensée maternelle jusqu'à des actions extrêmement larges comme la conception de systèmes publics d'éducation » (Tronto, 2012, 31). En s'inscrivant dans ce cadre, la recherche a questionné la possibilité du projet - à l'échelle de l'architecture et de l'urbain- de porter une contribution à une politique territoriale, multiscalaire et interdisciplinaire, pour passer de la gestion du risque à la résilience permanente.

La notion de care, parfois réduite au soin, a été ici dépassée par l'inclusion des corps, des objets, des environnements et des actions de reliance (Bolle de Bal, 1981) produites dans des systèmes complexes. Ainsi, à la logique de soin qui intervient a posteriori, dans une situation où aucune autre solution n'est plus possible (renvoyant à une résilience post traumatique), se substitue celle d'un care dialogique et, sinon prédictif, au moins capable d'identifier les lignes à suivre pour une sollicitude et une résilience permanente. Le care se présente ainsi à la fois comme une disposition, une intention qui induit un processus et comme une action, une pratique, ou même un projet. En effet, selon la philosophe américaine, le care est à la fois une construction culturelle et un travail tangible qui permet de créer une forme d'abduction: la satisfaction d'un besoin, passe nécessairement par la mise en place de dispositifs qui répondent aux attentes des individus. Comme l'écrit Tronto : « Le care nous imposant de réfléchir concrètement aux besoins réels et d'évaluer de quelle façon il peut leur être répondu, il introduit un certain nombre de questions sur ce que nous valorisons dans la vie quotidienne » (Tronto, 2009, 262).

A partir de ces observations, il est possible de mettre en place un dispositif d'analyse de la capacité de résilience d'un projet du care (par rapport à un projet urbain qui se donne comme objectif l'application de la solution technique isolée). Le dispositif d'analyse du projet est fondé sur l'adaptation des termes qui caractérisent la société du care selon Tronto :

- Un care établissant une relation horizontale avec l'environnement et l'idée de Nature ;

- Un care non exclusif avec une distribution des responsabilités (public/privé);

- Un care contextuel qui refuse l'essentialisme et inclut l'interculturel.

Le projet qui met en œuvre ce questionnement devient donc à la fois un levier pour encourager la réponse aux questions par des actes (actions) et un support pour co-concevoir, fabriquer ensemble, 
recycler la ville et partager les connaissances et les expériences. Le laboratoire urbain (Joseph, 1993) devient ainsi le terrain d'observation des synergies possibles entre ces stratégies du care.

\subsection{Complexité et énergie de la ville}

Nous insistons ici sur notre inscription dans une logique d'écologie urbaine (Coutard, Levy, 2010), dont le facteur central à reconsidérer est l'influence des logiques culturelles et interculturelles, trop souvent écartées, face à des manières technologiques de résoudre les problèmes environnementaux. Ces logiques culturelles se matérialisent par des comportements, des éléments de patrimoine, les aménagements végétaux, le partage des espaces publics et tous les facteurs souvent considérés comme des éléments de l'espace urbain. Les interactions, synergies et latences d'énergie, renvoient à l'existence d'une régulation complexe qu'on peut appeler la thermodynamique urbaine. Cette position implique une acceptation de la ville en tant que système complexe et, plus particulièrement, comme un ensemble de systèmes : systèmes énergétiques, technologiques, sociaux, économiques, culturels, informationnels et noologiques en interaction continue à beaucoup d'échelles spatiales et temporelles différentes (Prigogine, Stengers, 1979; Bocchi, Ceruti, 1985). Dans le laboratoire urbain, la complexité de l'échantillon et son inscription dans un complexe émergétique ${ }^{2}$ (Odum, 1996; Georgescu-Roegen, 2003), met en évidence l'importance des interactions du système anthropique, autrement dit tout ce qui échappe à la quantification matérielle des flux et qui relève d'échanges parfois non quantifiables, échelonnés sur une durée longue et s'activant selon des convergences de facteurs non planifiables. Dans notre cas d'étude, l'entrée par la lecture anthropique nous semblait indispensable car les déclencheurs des actions complexes sont bien les individus dans leur complexité ; si cette complexité est ramenée à une seule variable à la fois, les acteurs de l'énergie (essentiellement les usagers) sont réduits à des « profils de consommation » et, face à des innovations, produisent des feedback décevants pour les experts (Alessandrini et alii, 2014).

Dans la logique de la complexité, la représentation globale est compliquée (Morin, 1974 ; Varela, 2017) ; mais, en considérant un sous-système (macro-méso-micro) comme le reflet d'un système plus large, à la fois illustrant son comportement et étant le support des relations intrasystémiques, même un échantillon (en l'occurrence un croisement de rues et les immeubles, équipements, transports contigus observés par le biais de l'ICU) donne lieu à une observation transposable dans d'autres lieux (à la condition de la considérer comme un processus et non comme un modèle). Les villes sont ainsi caractérisées par une omniprésence du changement et une coévolution continue avec leurs habitants qui transforme leur forme matérielle (Salat, 2011).

\subsection{Projet et design des milieux}

La forme de la ville repose sur une capacité à faire cohabiter les réseaux et le système ponctuel de production d'énergie, ce qui implique une manière renouvelée de voir la «forme de la ville » en tant que possibilité de tous ses espaces (pleins, vides dans les parties visibles, mais aussi les sous-sols et les parties aériennes considérées comme des entités) de contribuer à la régulation climatique. Les différentes parties composent un milieu habité (Younès, Goetz, 2010) dont la qualité est une capacité d'adaptation permanente qui tend à une résilience constante et multipartenariale, ouverte à la participation des «urbains». Cet équilibre, dynamique mais continu, peut être maintenu par un ensemble de projets, un dispositif de projets, qu'on peut assimiler à une action de design des milieux. Les tensions qui parcourent le territoire sont dues à une imbrication de l'élément naturel, économique, social, anthropologique, mais, au lieu de créer des fractures et parcellisations, elles peuvent être entendues comme l'élément de reliance qui guide la logique de tissage que des micro-projets provoquent et qui ont trait au milieu urbain/vivant (Tufano, 2015). Or, si les processus de projet ne se réduisent pas à des simples productions de formes et de solutions techniques, il serait possible d'éviter la rupture entre les causes et les effets qui se joue entre une technostructure énergétique à l'échelle macro et la perception des effets à celle micro (urbaine) ou encore plus de l'individu (Gras, 1993).

\footnotetext{
${ }^{2}$ Le terme "émergie" correspond à embodied energy notion théorisée par Odum en 1996. 
Comme le résume l'économiste Paolo Perulli (2009), aux formes spatiales modernes se substitue un territoire à la densité tantôt distendue tantôt excessive, un espace discontinu, où les objets semblent suspendus et déconnectés du tissu de la ville. Cette hypothèse n'est pas sans conséquences pour la lecture (ou la construction) de l'espace urbain avec des projets innovants (Quenault, 2015). Dès lors, est-il possible de refonder un espace urbain où des formes inédites, celles du care, permettraient d'intégrer un design des milieux, un système de projets, fabriquant la résilience urbaine?

Comme le rappelle Terrin, il peut s'agir d'une conception par opportunités qui est différente de la gestion du risque et, en même temps, qui ne repose pas simplement sur la responsabilité individuelle, mais qui se structure comme une médiation par le projet où les récurrences projectuelles permettent de fixer les scénarios les plus pertinents et efficaces (Gascon, Terrin, 2016).

\section{Par le care : un système de projets pour accompagner la résilience.}

\subsection{Le contexte de la recherche : l'énergie de la ville}

Le Plan Local de l'Energie (PLE) de Paris, qui donne des contraintes particulières et contextuelles au cadre réglementaire national de la Loi de Transition Energétique, le SRCAE et, au niveau local, des Plans Climat Energie Territoriaux ${ }^{3}$ sont, dans l'ensemble, le cadre à l'échelle urbaine (macro) choisi pour l'inscription de la recherche. L'approche par l'ICU n'est qu'une échelle micro-méso qui permet de parler d'énergie, d'environnement et de risque en tant que catégories générales mais aussi en tant qu'entités matérielles, visibles, sensibles se prêtant à des expérimentations de projet (Raineau, 2012 ; Raineau, 2014). Le terrain de l'étude se situait dans le quartier de Jussieu (Paris $5^{\mathrm{e}}$ arrondissement) ; la co-présence de plusieurs éléments (patrimoine, forte circulation pendulaire, grands réseaux de transport, diversités des bâtiments, présence diverse de «nature en ville» et - au regard de l'échantillon choisi- diversité sociologique) permettaient une triple analyse :

- environnementale : objective et biocentrique ;

- urbaine : subjective/collective et anthropocentrique ;

- structurelle : technocentrique (infrastructures de mobilités et polarités liées à la vie collective).

Cette analyse renvoyait aux trois critères de l'urban caring (relation éco-logique, interculturelle et co-production des dispositifs techniques) et pouvait se décliner par des propositions de projet (spatial et immatériel).

\begin{tabular}{|c|c|c|c|}
\hline Année & Objectif & Type d'action & Acquis pour la phase suivante \\
\hline 2013 & $\begin{array}{l}\text { Rendre visible l'énergie } \\
\text { contenue dans le vivant (les } \\
\text { éléments du milieu qui rentrent } \\
\text { dans le processus de } \\
\text { métabolisme urbain organique) }\end{array}$ & $\begin{array}{l}\text { Workshop de projet entre écoles } \\
\text { d'architecture, d'art, avec } \\
\text { l'accompagnement de l'INRA. }\end{array}$ & $\begin{array}{l}\text { Rédiger le cahier des charges de la } \\
\text { phase suivante, en introduisant une } \\
\text { notion de prise de connaissance et } \\
\text { prise de conscience de l'aspect } \\
\text { sensible et sensoriel de l'énergie. }\end{array}$ \\
\hline 2014 & $\begin{array}{l}\text { L'impact climatique induit par } \\
\text { les îlots de chaleur urbains et sa } \\
\text { capacité à être un levier de } \\
\text { projets pour la résilience }\end{array}$ & $\begin{array}{l}\text { - Enquêtes } \\
\text { - Observation de terrain sur les } \\
\text { comportements urbains } \\
\text { - Recensement des matériaux de } \\
\text { l'espace public et des façades } \\
\text { - Analyse bâtiment par bâtiment des } \\
\text { caractéristiques au regard de l'énergie }\end{array}$ & $\begin{array}{l}\text { - Fiches du potentiel énergétique de } \\
\text { chaque bâtiment } \\
\text { - Mise en forme de plusieurs coupes } \\
\text { urbaines montrant les échanges entre } \\
\text { flux } \\
\text { - Scénarios de comportements à } \\
\text { l'échelle urbaine }\end{array}$ \\
\hline
\end{tabular}

${ }^{3}$ APUR, «Un Plan Local Energie (PLE) pour Paris et la métropole », dans Note, octobre 2014, n 81, p.1

(c) 2017 ISTE OpenScience - Published by ISTE Ltd. London, UK - openscience.fr 


\begin{tabular}{|l|l|l|l|}
\hline & $\begin{array}{l}\text { Hypothèse du care pour } \\
\text { l'intervention de projet dans des } \\
\text { coupes (transects) }\end{array}$ & $\begin{array}{l}\text { - Travail accompagné par des } \\
\text { spécialistes des énergies sur les formes } \\
\text { innovantes de l'énergie } \\
\text { Identification de trois transects } \\
\text { emblématiques des formes urbaines et } \\
\text { proposition de projet } \\
\text { Évaluation croisée par ELEMENTS } \\
\text { INGENIERIE et l'équipe de recherche }\end{array}$ & $\begin{array}{l}\text { - Construction d'outils de visualisation } \\
\text { et comparaison des projets entre } \\
\text { approche classique et projets : } \\
\text { d'interface, rythme, connexion } \\
\text { - Scénarios de résilience }\end{array}$ \\
\hline $\mathbf{2 0 1 6}$ & $\begin{array}{l}\text { Propositions spatiales pour le } \\
\text { logement }\end{array}$ & $\begin{array}{l}\text { Travail de design et projet d'innovation } \\
\text { Approche psycho-environnementale } \\
\text { Lopez, 2014; Charras, Eynard, 2014 }\end{array}$ & $\begin{array}{l}\text { Projets de mutualisation à l'échelle de } \\
\text { l'immeuble } \\
\text { Design d'éléments biomimétiques }\end{array}$ \\
\hline $\mathbf{2 0 1 7}$ & $\begin{array}{l}\text { Représenter les synergies } \\
\text { urbaines }\end{array}$ & $\begin{array}{l}\text { Séminaire, workshop de projet, } \\
\text { exposition. }\end{array}$ & $\begin{array}{l}\text { Représentation des scalabilités } \\
\text { (Brenner, 2004) urbaines }\end{array}$ \\
\hline
\end{tabular}

Tableau 1. Déroulement général de la recherche sur l'énergie (cf.note 2). Les résultats exposés dans l'article portent sur les projets développés en 2015 et 2016.

La question des enjeux de l'ICU parisiens (Bigorgne, Mangold, 2014) a été donnée comme clé d'entrée dans le projet, car elle incarnait une possibilité de travail de projet entre préconisations théoriques et formes matérielles qui a été rapidement saisie par les acteurs opérationnels de la ville, comme les agences d'urbanisme projet (Toubin, Faytre, 2015 ; Dycha, 2015). Nous nous sommes appuyés sur ces travaux avec l'objectif de comprendre les stratégies à développer par des scénarios de projet. Le travail à l'échelle méso était donc nécessaire car la rupture existante entre les impacts globaux et les pratiques locales semblait l'échelon à combler par le projet pour initier le processus de résilience permanente.

Les projets portent sur une série de modifications, souvent minimalistes, dont l'efficacité est accentuée par leur mise en réseaux, c'est-à-dire, par leur relation avec un système général programmé et accompagné par les acteurs institutionnels ou associatifs. Selon une approche abductive, inspirée par le travail de Tronto, nous avons envisagé les hypothèses possibles permettant de repérer, dans la phase d'étude et d'analyse, les données nécessaires aux expérimentations successives. Ainsi, un projet a été retenu pour les phases suivantes de la recherche s'il mettait en relation plusieurs niveaux : par exemple, si les solutions en matière de confort climatique dans le logement sont contraintes, un projet qui implique un prolongement dans les espaces ouverts de proximités est retenu comme « hypothèse de projet interscalaire » car il pourra à la fois jouer dans l'atténuation climatique à l'échelle méso, mais aussi offrir un espace individuel de confort qui prolonge à l'extérieur l'espace du logement (confort psychologique) et contribuer à la réduction de la consommation électrique domestique individuelle (Musy, 2015, 258). 


\begin{tabular}{|c|c|c|c|}
\hline $\begin{array}{l}1 \text { Comment } \\
\text { mesurer le } \\
\text { différentiel entre } \\
\text { les différentes } \\
\text { morphologies? }\end{array}$ & $\begin{array}{l}3 \text { Peut-on récolter et } \\
\text { recycler les énergies } \\
\text { dispersées dans l'urbain? }\end{array}$ & & $\begin{array}{l}\text { MICRO-MESO } \\
\text { Identifier pour les } 3 \\
\text { morphologies (linéaire, } \\
\text { compacte, autour de la place) } \\
\text { les éléments de typologie } \\
\text { architecturale à faire varier } \\
\text { respect des contraintes } \\
\text { patrimoniales pour réduire la } \\
\text { consommation }\end{array}$ \\
\hline $\begin{array}{l}4 \text { Comment } \\
\text { mesurer le } \\
\text { différentiel entre } \\
\text { les différentes } \\
\text { morphologies? }\end{array}$ & $\begin{array}{l}4 \text { Comment mesurer le } \\
\text { différentiel entre les } \\
\text { différentes morphologies } \\
\text { ? Les éléments de façade } \\
\text { et de toiture des } \\
\text { immeubles ont un rôle } \\
\text { d'interface entre l'échelle } \\
\text { de l'édifice et la ville } \\
\text { (confrontation typologie- } \\
\text { morphologie) } \\
5 \text { Comment trouver une } \\
\text { morphologie sobre dans } \\
\text { une ville dense? } \\
\text { La morphologie urbaine a } \\
\text { un impact sur les } \\
\text { températures urbaines }\end{array}$ & & $\begin{array}{l}\text { MICRO-MESO } \\
\text { Identifier les éléments et les } \\
\text { lieux de dispersion des } \\
\text { énergies. } \\
\text { Pratiquer deux approches de } \\
\text { projet : } \\
\text { - à travers l'analyse } \\
\text { systémique de la morphologie } \\
\text { (meso), on monte en échelle, } \\
\text { vers le macro ; } \\
\text { - à partir des points de } \\
\text { criticité, on intervient à } \\
\text { l'échelle micro }\end{array}$ \\
\hline $\begin{array}{l}2 \text { Peut-on } \\
\text { transformer des } \\
\text { immeubles de la } \\
\text { ville dense en } \\
\text { édifice à } E+? \\
\text { Les éléments de } \\
\text { façade, toiture et } \\
\text { les caves } \\
\text { deviennent des } \\
\text { lieux de } \\
\text { production et } \\
\text { stockage de } \\
\text { l'énergie. }\end{array}$ & $\begin{array}{l}1 \text { Comment mesurer le } \\
\text { différentiel entre les } \\
\text { différentes morphologies } \\
\text { ? Les éléments de façade } \\
\text { et de toiture des } \\
\text { immeubles ont un rôle } \\
\text { d'interface entre l'échelle } \\
\text { de l'édifice et la ville } \\
\text { (confrontation typologie- } \\
\text { morphologie) }\end{array}$ & $\begin{array}{l}3 \text { Peut-on récolter et } \\
\text { recycler les énergies } \\
\text { dispersées dans } \\
\text { l'urbain? } \\
\text { Les lieux immobiles } \\
\text { de la mobilité et les } \\
\text { interstices urbains } \\
\text { peuvent être un } \\
\text { levier de changement } \\
5 \text { Comment trouver } \\
\text { une morphologie } \\
\text { sobre dans une ville } \\
\text { dense? }\end{array}$ & $\begin{array}{l}\text { MICRO-MESO-MACRO } \\
\text { Identifier pour les } 3 \\
\text { morphologies (linéaire, } \\
\text { compacte, autour de la place) } \\
\text { des éléments de typologie } \\
\text { architecturale à faire varier ; } \\
\text { respect des contraintes } \\
\text { patrimoniales pour réduire la } \\
\text { consommation- } \\
\text { Croiser les données } \\
\text { sociologiques et les potentiels } \\
\text { d'innovation }\end{array}$ \\
\hline
\end{tabular}

\section{Environnementale}

\section{Structurelle}

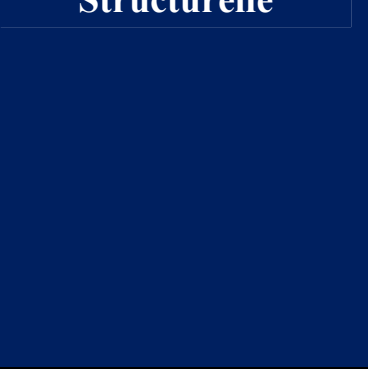

Urbaine

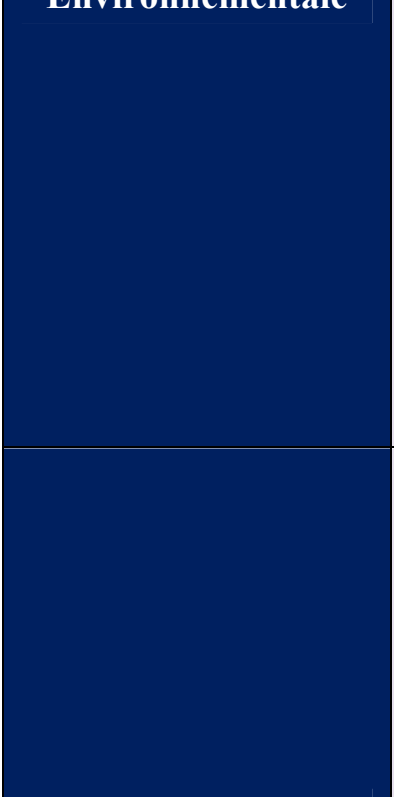

Tableau 2. Réalisé en 2015, après le répertoire des matériaux présents dans notre secteur de travail, ce tableau a servi à identifier les éléments qui manquaient pour compléter le répertoire matériel de l'urbain et à mettre en place des hypothèses sur les relations entre projet spatial/matériel-comportement induit. 


\subsection{Description du dispositif et des variables de projet à introduire}

A partir du tableau d'hypothèses (points 1 et 2 du tableau 2), en utilisant la base d'informations SIG fournie par l'Atelier Parisien d'Urbanisme et en l'augmentant d'observations de terrain, des fiches ont été réalisées pour identifier les potentiels énergétiques de chaque bâtiment.

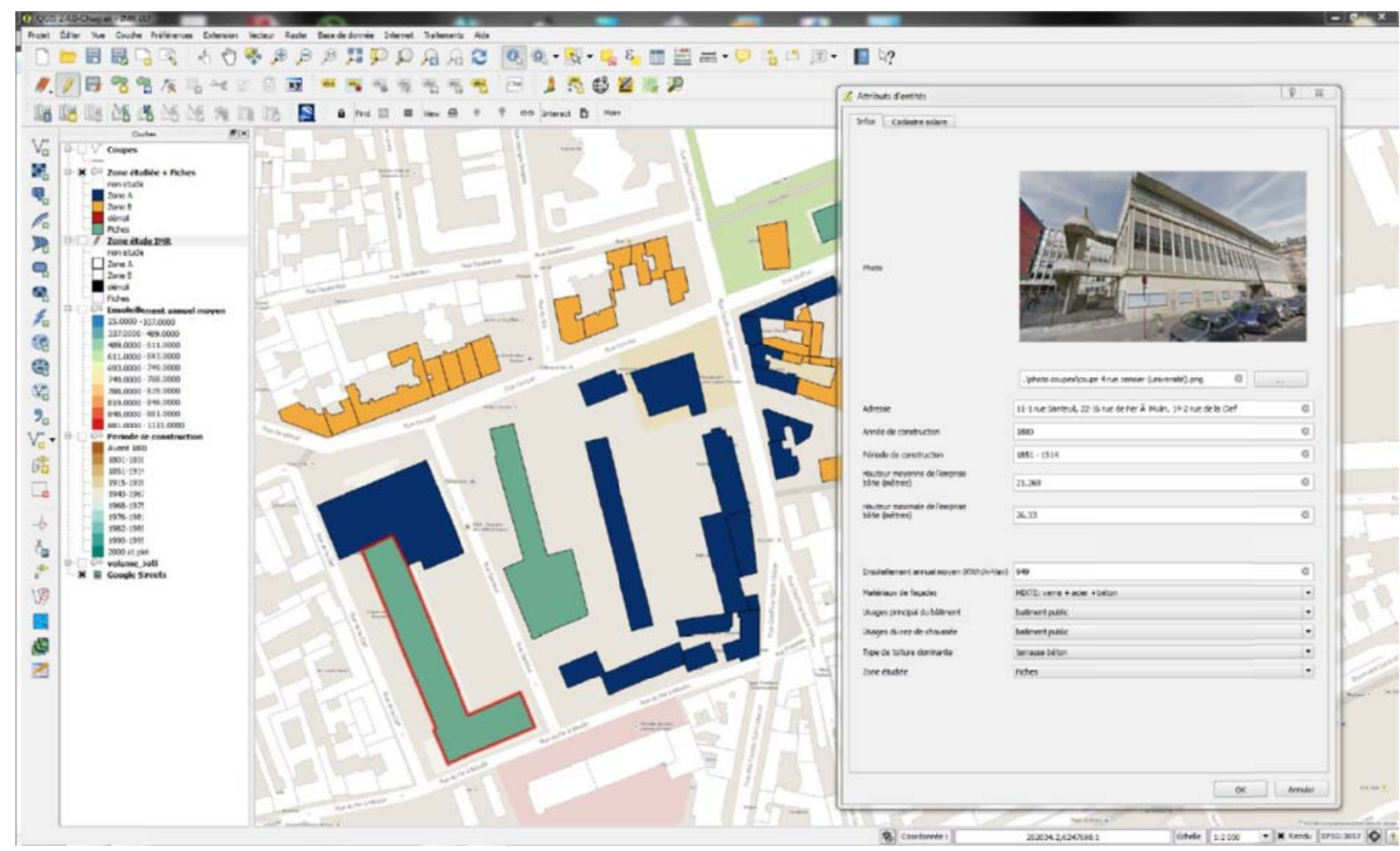

Figure 2. Pour chaque bâtiment, outre l'ancienneté, l'emplacement et la taille (emprise au sol, hauteur), ont été précisés : le stock bâti (âge du bâti, densité du bâti, typologie du bâti, statut de la propriété, densité de population, ménages à bas revenus) ; les éléments de façade et les matériaux ; les matériaux de la couverture et le potentiel d'ensoleillement ; la présence de caves ; la distance des bâtiments environnants ou, le cas échéant, la mitoyenneté. Le dernier volet déroulant de la fiche présente une évaluation des éléments qui composent les enveloppes (albédo, porosité des matériaux, effet venturi...).

Après avoir répertorié les immeubles, il a été question de choisir des échantillons en fonction des morphologies urbaines, des usages et de la mobilité. A partir de ces échantillons considérés comme les types morphologiques qui caractérisent l'arrondissement, a été effectué un double travail à l'échelle meso (hypothèses 3 et 4 du tableau 2) : d'une part, un travail de reconnaissance des matériaux des espaces ouverts (publics et- dans la mesure du possible- privés), permettant de compléter les informations fournies par les fiches sur les bâtiments; d'autre part, un travail d'observation des fréquentations en fonction des usages et de l'importance de la mobilité, permettant d'établir des cartes identifiant les points sensibles pour les vulnérabilités environnementale (points de chaleur; impact sonore ; zone particulièrement exposées au vent en période hivernale...). 


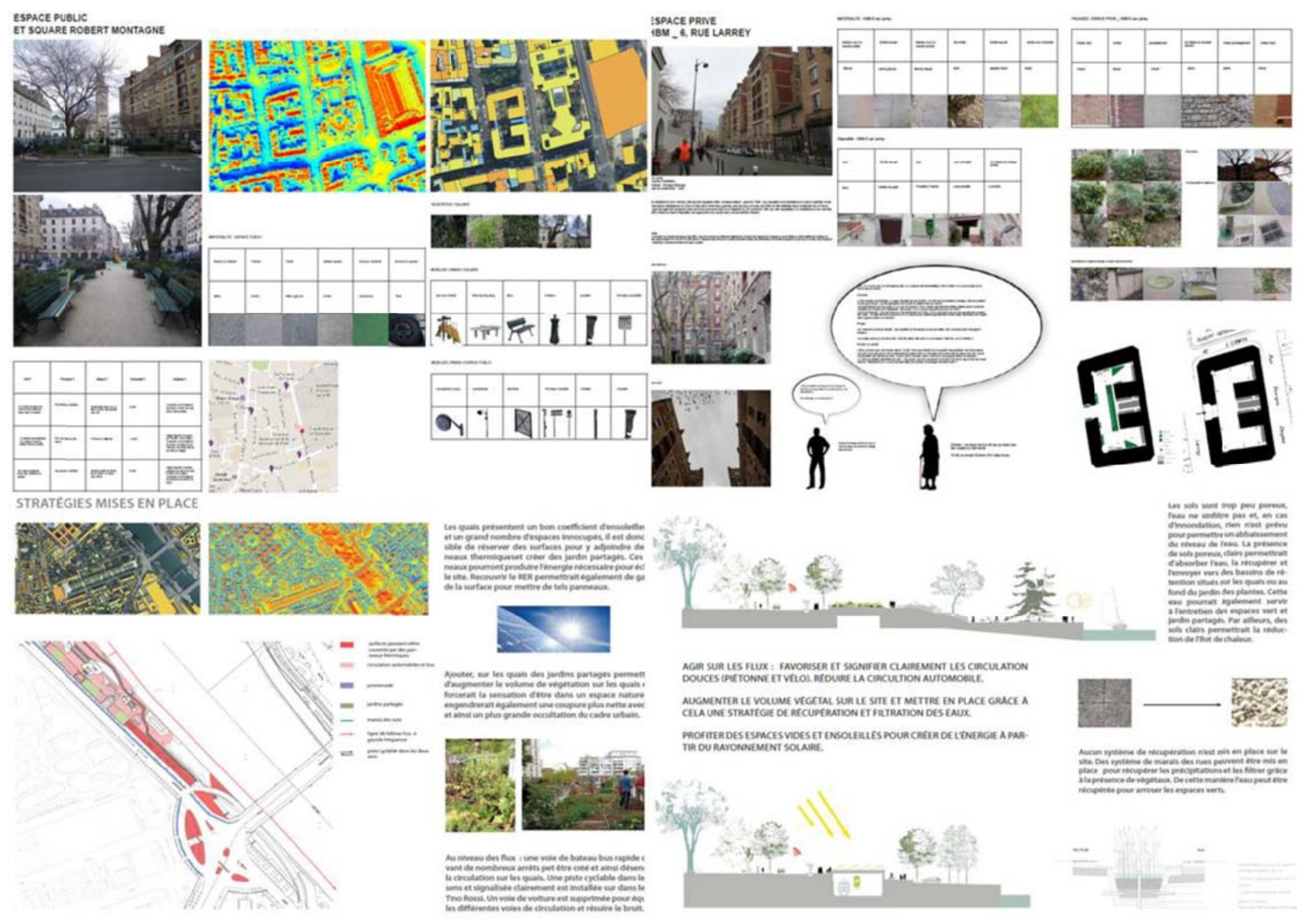

Figure 3. Exemple d'une planche de synthèse des analyses urbaines.

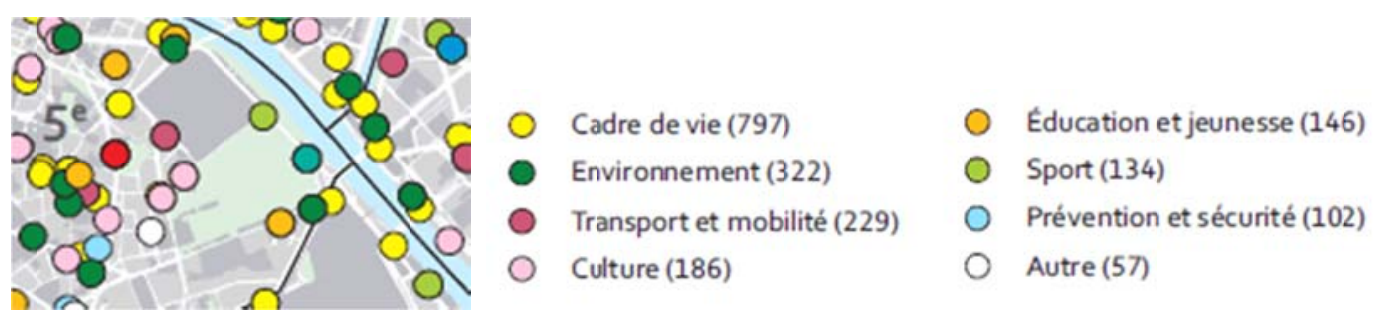

Figure 4. Carte des projets portés dans le cadre du budget participatif de la ville de Paris. Les lieux où se situent des activités associatives relevant de la culture, de l'environnement et de l"éducation ont été observés de manière prioritaire pour identifier le public susceptible d'initier des activités de sensibilisation sur la relation énergie, espace naturel, partage de sociabilité. Source : Ville de Paris, APUR, 2015

Des fiches de criticité ont permis d'établir un diagnostic et d'identifier les actions qui auraient pu accompagner la rénovation énergétique de l'enveloppe du bâtiment par des projets de partage et de mise en relation, selon les préconisations de l'urban caring. Face à la difficulté de pouvoir tout embrasser, nous avons préféré donner des indications de projet à réaliser à travers des critères qui mettent en œuvre la notion de mutualisation des énergies (via les réseaux et la sensibilisation à la modulation des consommations en fonction des heures de la journée). Ces critères sont :

- l'innovation technique inspirée d'un bio-design ;

- l'innovation architecturo-programmatique faite « avec»l'habitant et l'existant;

- la porosité des milieux habités entre humain et non humain, naturel et artificiel ; 
- la multiplication des projets d'espaces d'association, mutualisation, coopération...;

- l'accessibilité à différentes facilités (mobilités, équipements...) ;

- la coproduction public-privé.

Le projet a été développé selon la logique de la coupe à l'échelle urbaine, le transect (Duany, 2002), qui permet de visualiser, comprendre et mettre en synergie les éléments visibles et non visibles, matériels et immatériels (Addington, 2008). En tout, trois transects ont été choisis et sur chacun il y a eu deux hypothèses de projet faites à partir des six critères. Le résultat a ensuite été soumis à l'expertise d'un cabinet d'ingénierie pour effectuer les évaluations énergétiques en comparant les résultats d'une réhabilitation "classique» proposée par le cabinet d'ingénierie et celle des projets d'innovations réalisés dans le cadre de la recherche.
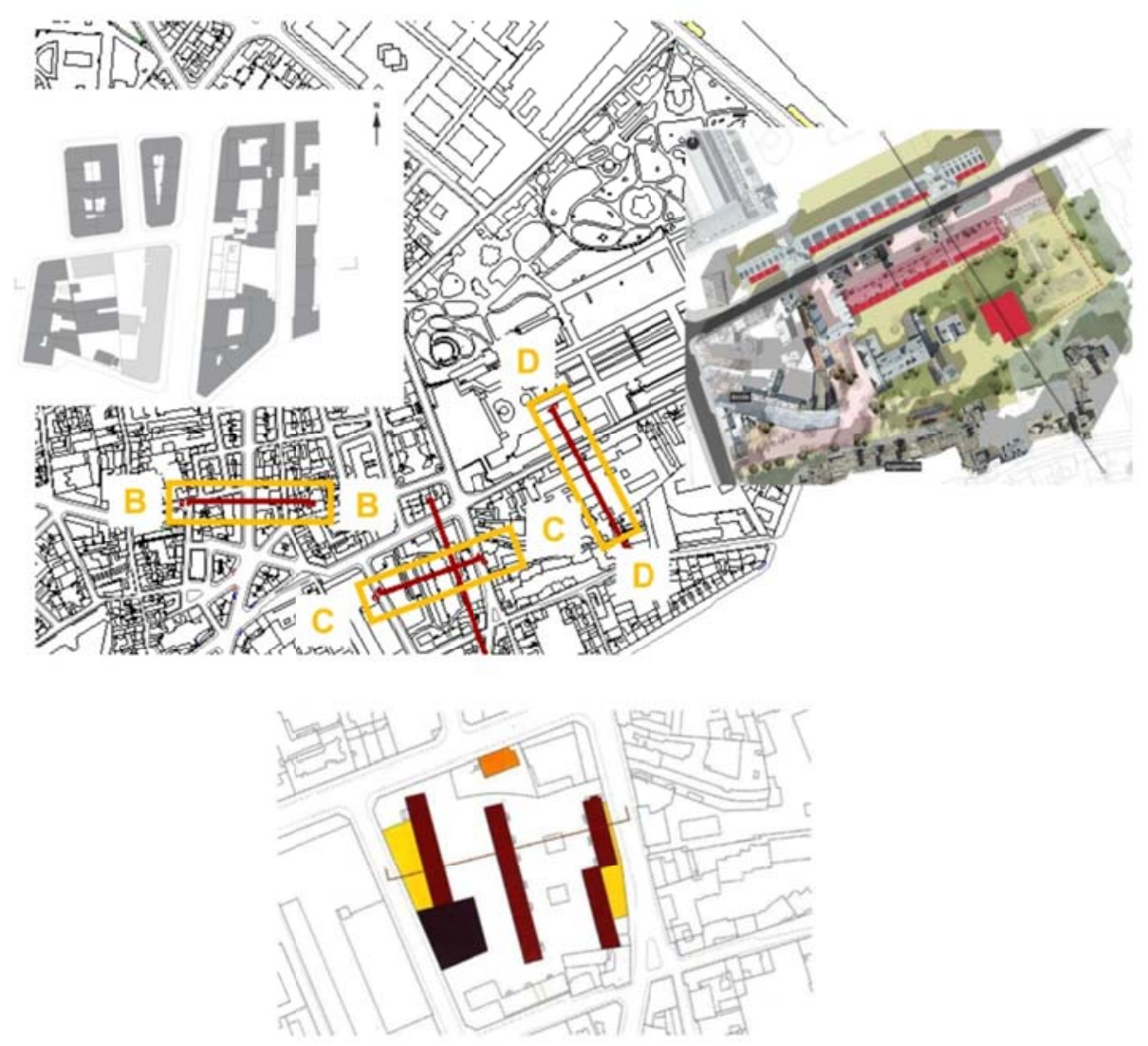

Figure 5. Identification des trois transects qui ont fait l'objet de l'évaluation du cabinet d'études Eléments Ingénierie (Sophie Moreau et Aurore Pfiszter). La coupe B-B se situe rue de Monge, la coupe C-C sur le groupe de logements donnant sur la rue Santeuil, la coupe D-D, recoupe des bâtiments datant du XIXe siècle, vétustes, situés rue Buffon. Par leurs statuts et fonctions différents, ces transects permettaient d'envisager des scénarios d'usage divers (B-B, immeuble en copropriété construit dans les années 70 ; C-C immeuble locatif social construit dans les années 60 appartenant au bailleur Paris Habitat; D-D, les laboratoires du Museum, à usage tertiaire) et représentaient également des morphotypologies urbaines rentrant dans les cas analysés par l'APUR (Bigorgne, 2014).

Les projets ont été analysés en intégrant notamment les réflexions quant aux gisements énergétiques urbains $^{4}$. En simplifiant certaines approches, pour établir des tendances, et en faisant une moyenne entre l'évaluation des scénarios d'utilisation, à l'échelle de l'îlot, et la moyenne thermique par bâtiment, le cabinet d'études a essayé d'effectuer l'analyse des propositions de projet en intégrant une

\footnotetext{
${ }^{4}$ Un séminaire de travail a réuni plusieurs chercheurs et praticiens qui ont présenté les potentialités du territoire (sol et sous-sol) parisien : Julien Bigorgne (APUR), Mathieu Fernandez (CNAM, HT2S), Véronique Merrien (CNAM, département de géologie), Sophie Moreau (ELEMENTS INGENIERIE). 
marge d'incertitude associée à certaines ressources énergétiques (réseau des eaux usées, chaleur du métro etc.). ${ }^{5}$ Les propositions des équipes de projet ont surtout préconisé la mise en œuvre de systèmes innovants de production et/ou de récupération énergétique, ce qui permet de réduire la consommation en énergie primaire, mais qui souvent ne réduit pas intrinsèquement la demande de chauffage. Elles présentaient en revanche l'intérêt d'une démarche inventive à l'échelle d'un quartier en travaillant sur la mutualisation et la récupération. Les propositions de production locale (photovoltaïque) permettent de compenser d'ailleurs une partie de la consommation.

La comparaison montre un différentiel faible entre un projet classique de réhabilitation énergétique et un projet innovant fondé sur les six critères. En effet, les analyses réalisées ne pouvaient pas considérer une variable temps plus longue, permettant d'amortir les coûts, et d'autres critères d'évaluation, comme la compensation des effets climatiques en relation avec les usages de l'énergie ou les formes de confort psychologique (usage et partage dans les espaces publics).

Les résultats des simulations énergétiques ont mis en évidence que la discriminante principale affectant la distribution temporelle des besoins thermiques et sa valeur totale est l'intensité énergétique des charges internes liées aux utilisations prévues. À l'échelle d'un bâtiment, les conditions environnementales sont modifiées moins par les effets de l'îlot de chaleur au niveau urbain, qui se répercutent sur les caractéristiques météorologiques citadines, que par les conditions microclimatiques des espaces ouverts autour du bâtiment qui en altèrent les températures effectives d'échange et les états thermodynamiques de l'air de ventilation. Le projet à l'échelle du bâtiment devient donc un pivot de l'espace urbain et de ses fonctions (interface intérieur-extérieur/ surface-sous-sol/enveloppe-air).

Les espaces ouverts et les interstices, en raison de leur plus grande flexibilité (morphologique et d'usage) par rapport aux pleins (les bâtiments), peuvent jouer un rôle déterminant dans le processus de renaissance environnementale des villes qui accompagne la résilience (Murer, 2014 ; APUR, 2017). Ce changement de posture induit par l'appréhension de la «physique de la ville»-notamment les flux rendus visibles par les Ilots de Chaleur Urbains et comptés comme des matières à projet- demande au concepteur une proposition de projet pensée plus en termes d'amélioration que de création. Cette inversion de la figure du concepteur, de démiurge à accompagnateur de projets, implique une forme de partage du projet, de relationnalité nécessaire, comme le souligne Tronto, qui renvoie à une créativité élargie dont les usagers peuvent être les acteurs. La réalisation des interventions complexes permet d'ultérieures réductions des besoins énergétiques qui se reflètent également à l'échelle du quartier, ce qui réduit l'empreinte énergétique. Plus que de performance, il s'agit d'une " capacité énergétique » plus complexe à appréhender mais plus apte à s'adapter aux différents contextes et situations habitées. Ainsi, la réussite d'une opération en termes de «capacité à la résilience » ne repose pas sur la superposition des opérations, mais sur une amélioration des conditions d'habiter où l'occupant est activement impliqué et dont les implications sont globales (Bigorgne, 2016).

Pour pouvoir imaginer les effets d'un scénario à plus long terme et ses bénéfices, trois figures ont été identifiées comme celles qui montraient le plus grand potentiel de relationnalité :

- l'interface, entendue comme relation entre le bâti et l'espace extérieur végétal, individu/espace d'habitation/espace interstitiel/espace de proximité ;

- les connexions entre les usages et le potentiel morphologique ou encore entre l'entité privée (logements appartenant à Paris Habitat) et ceux publics ou semi publics (établissements universitaires ; parking dans le sous-sol de l'îlot) ;

- les rythmes, les temporalités, et, notamment, l'inversion de cycle entre les usages domestiques (concentrés dans la tranche horaire 17h-23h et 6h-8h) et ceux des édifices publics (de 9h à 16-17h).

\footnotetext{
${ }^{5}$ Les simulations ont été réalisées au moyen du logiciel Design Builder (moteur de calcul Energy+) qui modélise en 3D les bâtiments et étudie leur comportement thermique par pas de temps. 
Ainsi, s'il est possible de conclure que les solutions inventives, faites à partir des figures de partage, sont comparables à celle standard, pour activer un dispositif qui accompagne la résilience urbaine il faut aller au-delà du projet ponctuel et intégrer trois modifications dans la manière de faire et fabriquer le projet :

- le projet de l'édifice ne doit pas être conçu de manière centripète, c'est-à-dire comme le point de convergence de toutes les solutions techniques isolées, mais plutôt comme le point de rayonnement et d'interface de l'intérieur à l'extérieur ;

- le projet urbain où se situe le bâtiment ne peut pas être pensé « en plan » mais à travers la notion de transect qui permet de visualiser les connexions (flux) et de les transformer en une matière à projet où l'édifice s'insère ;

- les fonctions et les usages doivent être pensés de manière prospective, c'est-à-dire non seulement en les intégrant dès le début par la programmation et la co-conception, mais aussi dans leur développement temporel et par la possibilité de créer des rythmes alternési (travail-logement, loisiréducation...).

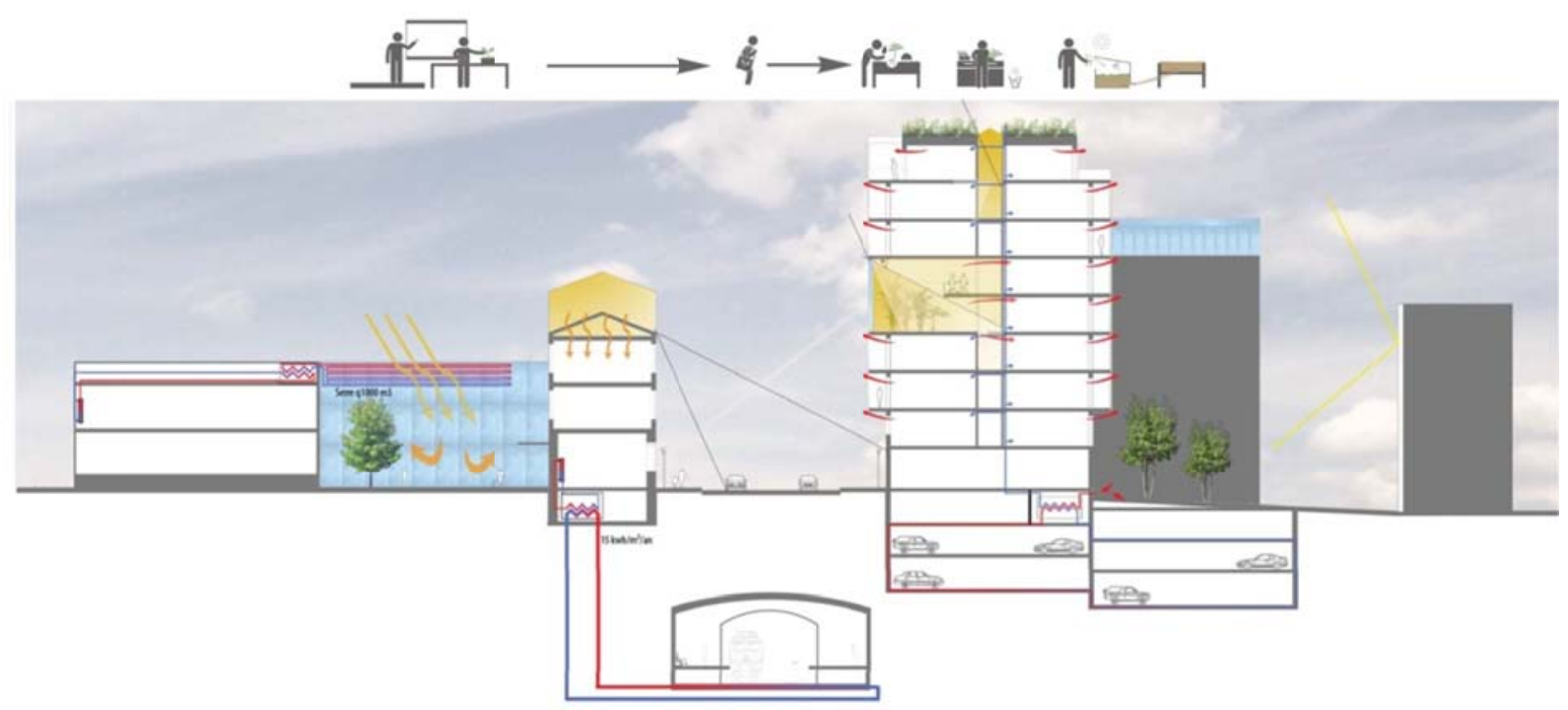

Figure 6. Un projet de connexion et interface. Le projet proposé dans l'immeuble de la rue Monge, en coupe, permet de visualiser les mutualisations possibles avec les réseaux souterrains et avec une exploitation temporelle différente du bâtiment scolaire situé sur l'autre trottoir. La proposition d'une serre (projet de photosynthèse urbaine) initie des actions de sensibilisation aux cycles courts partagées par les habitants de l'immeuble et accompagnées dans le cadre des activités de l'école. La limite de ce projet a été identifiée avec : la difficulté à initier des activités collectives dans une copropriété présentant une forte résistance à l'acceptation d'éléments naturels dans les parties communes ou en toiture (entretien, risque de présences animales ....). 


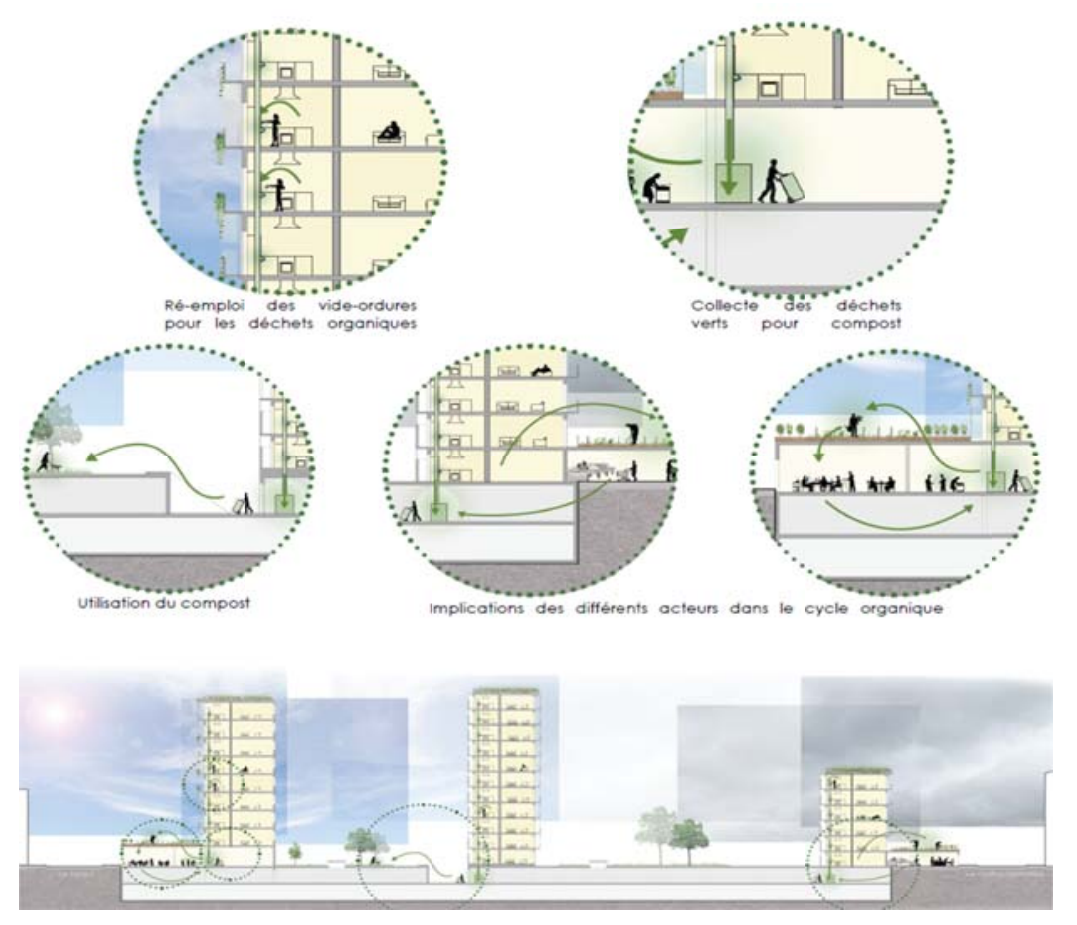

Figure 7. Un projet d'interface. Le projet en coupe sur les immeubles de Paris Habitat (rue Santeuil, 5e), joue essentiellement sur la notion de socle et remet en activité les parties non visibles des logements. Dans ce cas de figure, le projet a surtout servi comme « agent de médiation et sensibilisation » auprès des habitants sollicités pour avoir leur avis sur l'investissement individuel dans la question de la ville sobre.

\section{Conclusion}

Dans notre cas d'étude, situé dans le $5^{\mathrm{e}}$ arrondissement de Paris, dans un contexte complexe- qui présente diverses morphotypologies urbaines, un système de mobilité multimodal, des services couvrant l'enseignement, le loisir et la culture, des commerces répondant aux demandes d'un échantillon sociologique très varié avec une dominante de personnes âgées seules et d'étudiants- nous avons émis l'hypothèse d'une possibilité d'initier un processus de résilience permanente à travers un dispositif de projets qui s'inscrivent dans la temporalité continue de l'urban caring (relations avec l'environnement; partage des responsabilité et co-production des projets ; interculturalité, au sens anthropologique et épistémologique). L'observation a été faite sur l'Ilot de Chaleur Urbain en tant que dispositif capable d'investir trois échelles (micro-méso-macro) en fonction des reliances initiées par des projets. Inscrites dans la problématique de l'ICU et essayant d'activer les actions de partage et coproduction du care, les propositions faites sur trois transects urbains ont été analysées au regard des critères d'évaluation énergétique classiques (normes; adaptation) et, en parallèle, à travers une formalisation des « figures du partage ». Un dispositif descriptif a identifié des qualités et propensions qui évoluent (et deviennent de plus en plus efficaces) en fonction de scénarios d'usage allant du simple au complexe (selon six variables : technique ; innovation ; impact social ; socio-économie ; confort ; comportements). Dans ce contexte, les espaces ouverts ont été identifiés comme le facteur d'ajustement le plus important, ainsi la notion d'ICU, loin de se résoudre à un simple dispositif d'amélioration des conditions climatiques, peut jouer un rôle central, de pivot, dans l'implémentation d'un urban caring. L'appui sur la notion de care a permis d'utiliser des dispositifs à l'échelle de l'individu ayant des effets sur les autres échelles par interface et connexions et, du méso au macro, par rythmes et connexions. Le projet matériel situé joue donc un rôle dans cet élargissement scalaire à la condition de dépasser le projet architectural (une réponse matérielle à une question posée) ou urbain (dont les problématiques se résument aux impératifs du durable) pour l'élargir à la notion de système de projets (design des milieux) pensé de manière inverse au projet classique à travers les figures de la connexion, du rythme et de l'interface. 


\section{Bibliographie}

AdDington M., «Energy, Body, Buiding : Rethinking Sustainable Design », dans SAUNDERS W.,Nature, Landscape and Building for Sustainability, Harvard Design Magazine Reader, 6, 2008 (University of Minnesota Press) pp. 157-168

Alessandrini J.-M., François C., Fromy Ph., GangneuX M. C.,, Hognon B., Nibel S., L'exigence énergétique entre contrainte et innovation, PUCA, février 2014.

APUR, «Les îlots de chaleur urbains à Paris », Cahier\#4 : influence climatique des revêtements de sol à Paris, Ville de Paris, 2017.

AUBERT N., " Urgence et instantanéité : les nouveaux pièges du temps », dans AsCHER F., GODARD F., Modernité : la nouvelle carte du temps, Aube-Datar, La Tour d'Aigues, 2003.

BARLES S., " Le métabolisme urbain et la question écologique », Annales de la recherche urbaine 92 (sept. 2002), pp. 143-157.

Barroca B., Serre D., Diab Y., Penser la ville et agir par le souterrain, Presses de l'Ecole nationale des ponts et chaussées, Paris, 2014.

BAUDOUÏ R. , « Penser la transition éthique de l'urbanisme pour l'aménagement de villes durables. Le cas de la France et de la Suisse », Éthique publique, vol. 16, n 1, 2014, URL :http://ethiquepublique.revues.org/1404

Bigorgne J., MANGOlD A., Les îlots de chaleurs urbains. Cahier 2 : simulations climatiques de trois formes urbaines, APUR, Paris, 2014, www.apur.org/sites/default/files/documents/ilots chaleur urbains paris cahier2.pdf

BIGORGNE J., « Réhabiliter, économiser l'énergie et assurer la résilience de la ville », JEUDY O., NUSSAUME Y.,

PERYSINAKi A.(dir.), Paysages urbains [parisiens] et risques climatiques, Paris, Archibooks, 2016, pp.146-156.

BoCCHI G., CERUTi M., La sfida della complessità , Feltrinelli, Milano, 2007 (1985).

BOLLE de BAL M., « Reliance : Connexions et sens », Connexions, 1981, n 33, pp. 9-36.

Boxenbaum E., Laurent B., Lacoste A., Nouvelles énergies pour la ville du futur, Presses des Mines, Paris, 2013.

BRENNER N., New state spaces : urban governance and the rescaling of statehood, Oxford University Press, 2004.

Chaline C., Dubois-Maury J., Les risques urbains, Armand Colin, Paris, 2004.

CHARRAS K., EYNARD C., « Penser un environnement pour l'accueil des personnes présentant des vulnérabilités sociales et psychologiques : de l'élaboration à la conception architecturale, MARCHAND D., DEPEAU S.,

WeISS K., L'individu au risque de l'environnement, InPress, Paris, 2014,pp.175-198

ChU E., GoH K., Global Ecologies: Politics, Planning, and Design, Projection, 11, MIT Department of Urban Studies and Planning, 2015.

CONAN, M., Concevoir un projet d'architecture. L'Harmattan, Paris, 1990

Coutard O., LeVy J.-P., Ecologies Urbaines, Economica-Anthropos, Paris, 2010.

Cros M., Gaultier-Gaillard S., Harter H., PeCh P., Catastrophes et risques urbains. Nouveaux concepts, nouvelles réponses, Lavoisier, Paris, 2010.

DAuphine A., Provitolo D., «La résilience : un concept pour la gestion des risques », Annales de Géographie, 654, 2 , 2007, pp. 115-125.

DuANY, A., "Transect Planning», Journal of the American Planning Association, Vol. 68, No. 3, Summer 2002, American Planning Association, pp. 245-246.

DYCHA C., «Construire au contact du risque c'est comprendre le risque », Bâtir au contact du risque, Philotope, 11, juin 2015, pp. 87-92.

FELLI R., "Adaptation et résilience : critique de la nouvelle éthique de la politique environnementale internationale», Éthique publique, vol. 16, n 1,2014, URL : http://ethiquepublique.revues.org/1371

Fischer B., Tronto J. , "Towards a Feminist Theory of Care », ABEl E., Nelson M., Circles of Care : Work and Identity in Women'sLives, Albany, State University of New York Press, New York, 1991.

GASCON E., TERRIN J.-J., «Gestion des risques et des opportunités dans les projets d'aménagement urbain », Jeudy O., Nussaume Y., Perysinaki A.(dir.), Paysages urbains [parisiens] et risques climatiques, Paris, Archibooks, 2016, pp. $57-63$ 
Georgescu-Roegen N., The Entropy Law and the Economic Process Entropy, Harvard University Press, Cambridge Mass., 2013

GRAS A., Grandeur et dépendance. Sociologie des macrosystémes techniques, PUF, Paris, 1993

JoSEPH I., « Du bon usage de l'école de Chicago », Roman J, Ville, exclusion et citoyenneté. Entretiens de la ville II, Paris, Éd. Esprit, 1993, pp. 69-96

Kennedy C., CUdDiHI J., Engel-Yan J., « The changing metabolism of cities», Journal of Industrial Ecology, 11, 2, 2007, pp.43-59.

LAUGIER S., Tous vulnérables. Le care, les animaux, l'environnement, Petite Bibliothèque Payot, Paris, 2012.

LE Moigne J.-L., «Edgard Morin, le génie de la reliance », Synergies Monde, n 4 - 2008 pp. 177-184

LOPEZ A., « La complexité sans complexe de la psychologie environnementale », dans MARCHAND D., DEPEAU S.,

WeIss K., L'individu au risque de l'environnement, InPress, Paris, 2014, pp. 61-86.

LUSSAUlT M.,L'homme spatial. La construction sociale de l'espace humain, Seuil, Paris, 2007.

REgHeZZA-ZitT M., RUFAT S., «L'adaptation en Île-de-France entre injonction et recyclage. Techniques et politiques de la société de l'incertitude », Développement durable et territoires [En ligne], Vol. 6, n 3 | Décembre 2015. URL : http://developpementdurable.revues.org/11035 ; DOI : 10.4000/developpementdurable.11035

MARIE J.-B., « Les paradoxes climatiques des villes européennes », TERRIN J.-J., Villes et changement Climatique. Ilots de chaleur Urbains, Parenthèses, Marseille, 2015, pp. 24-36

METZGER P., D’ERCOLE R., « Les risques en milieu urbain : éléments de réflexion », EchoGéo, 18, 2011

METZGER P., ROBERT J., " Environnement et risques : les sciences sociales piégées entre critique radicale et utilité sociale», Espaces et rapports de Domination, PUR, Rennes, 2015.

MONGIN O.,La ville des flux. L'envers et l'endroit de la mondialisation urbaine, Fayard, Paris, 2013.

Mostafavi M., DOHERTY G., Ecological urbanism, Lars Müller-Harvard University, 2010.

MURER P., La transition énergétique, Mille et une nuits, Paris, 2014.

Musso P., «Innovations et usages », dans MUSSO P. avec CROZET Y., JOIGNAUX G., Le territoire aménagé par les réseaux, Aube-Datar, La Tour d'Aigues, 2002.

MUSY M., «Les Ilots de Chaleur Urbains : changer de focale », TERRIN J.-J., Villes et changement Climatique. Ilots de chaleur Urbains, Parenthèses, Marseille, 2015, pp. 244-258

NAVARRO-CARRASCAL O., Michel-Guillou E., "Analyse des risques et menaces environnementales: un regard psycho-socio-environnemental », MARChAND D., DePEAU S., WeISS K., L'individu au risque de l'environnement, InPress, Paris, 2014, pp.271-298

ODUM, H.T.,Environmental Accounting: Emergy and Environmental Decision Making, John Wiley \& Sons, New York, 1996

PERULLI P., Visioni di città. Le forme del mondo spaziale, Piccola Biblioteca Einaudi, Torino, 2009.

Pivot C., RYCHEN F., La gestion des risques à l'horizon 2020, l'Aube-Datar, La Tour d'Aigues, 2003.

PRIGOGINE I., Stengers I., La nouvelle alliance. Métamorphose de la science, Gallimard, Paris, 1979.

RAINEAU L., «Innover autrement : la technique face à la crise environnementale », Habitat et transition énergétique, sous la direction de François Rochon, Paris, L’Harmattan, 2014, pp. 49-69

POIROT-DELPECH Sophie, RAINEAU Laurence, Socio-anthropologie de l'environnement (1). Par-deçà le local et le global, L’Harmattan, collection Logiques Sociales, Série SocioAnthropo-Logiques, Paris, 2012.

Prost, R., Pratiques de projet en architecture : le tournant silencieux. Infolio, Gollion, 2014

QUENAULT B., «La dialectique vulnérabilité/adaptation des villes au changement climatique comme potentiel destructeur/créateur : quelles perspectives pour un développement urbain durable ? », FOREST J., HAMDOUCH A., Quand l'innovation fait la ville durable, Presses polytechniques et universitaires Romandes, Lausanne, 2015, pp. 2144

SALAT S., LABBE F., NOWAKI C., Les Villes et les formes. Sur l'urbanisme durable, Hermann, Paris, 2011.

SAUNDERS W., Nature, Landscape and Building for Sustainability, Harvard Design Magazine Reader(University of Minnesota Press), 6, 2008. 
TERRIN J.-J., «Penser la ville avec le climat », TERRIN J.-J., Villes et changement Climatique. Ilots de chaleur Urbains, Parenthèses, Marseille, 2015, pp.10-23

TODD N., Eco-design : des solutions pour la planète, Ecosociété, Montreal, 2007.

TRONTO J., Un monde vulnerable. Pour une politique du care, La Découverte, Paris, 2009 (1993).

TRONTO J., Le risque ou le care, PUF, Paris, 2012.

TOUBIN M., FAYTRE L., «La résilience urbaine face aux risques : nécessité d'une approche collaborative », dans Note Rapide de l'IAU, 682, mai 2015. URL : http://echogeo.revues.org/12640

TOUbin M., Lhomme S., Diab Y., Serre D., LAGANiER R., « La Résilience urbaine : un nouveau concept opérationnel vecteur de durabilité urbaine ? ", Développement durable et territoires [En ligne], Vol. 3, $\mathrm{n}^{\circ} 1 \mid$ Mai 2012. URL : http://developpementdurable.revues.org/9208 ; DOI : 10.4000/developpementdurable.9208

TUFANO A., « Un laboratoire urbain de l'énergie: un quartier parisien entre patrimoine et innovation », dans Scarwell H.J., Groux A., Leduq D., Transitions énergétiques : quelles dynamique de changement ? L' Harmattan, Paris, 2015, pp. 175-185.

VARELA F., Le cercle créateur. Ecrits (1976-2001), Seuil, Paris, 2017

Walker B., Salt D., Resilience Practice. Building Capacity to Absorb Disturbance and Maintain Function, Island Press, Washington, Covelo, London, 2012.

Walker B., Salt D., Resilience Thinking. Sustaining Ecosystems and People in a Changing World, Island Press, Washington, Covelo, London, 2006.

Wisner B., Blaikie P., CANNON T., DAVIS, I., At Risk: natural hazards, people’s vulnerability and disasters, Second edition, 2003.

Younes C., Goetz B., « Mille milieux », Le Portique, 25, 2010, URL : http://leportique.revues.org/2471 